\title{
Philosophiques
}

\section{Un modèle général pour décomposer la relation d'impact dans les recherches sur l'impact social de la science et de la technologie}

\section{Normand Lacharité}

Volume 16, numéro 1, printemps 1989

URI : https://id.erudit.org/iderudit/027066ar

DOI : https://doi.org/10.7202/027066ar

Aller au sommaire du numéro

Éditeur(s)

Société de philosophie du Québec

ISSN

0316-2923 (imprimé)

1492-1391 (numérique)

Découvrir la revue

Citer cet article

Lacharité, N. (1989). Un modèle général pour décomposer la relation d'impact dans les recherches sur l'impact social de la science et de la technologie.

Philosophiques, 16(1), 109-147. https://doi.org/10.7202/027066ar

\section{Résumé de l'article}

L'impact social de la science et de la technologie se manifeste par une série de relations que le présent modèle va découper sur des boucles de rétroaction. L'activité techno-scientifique des humains crée des perturbations dans le réseau des relations interindividuelles et sociales ainsi que dans l'environnement physique ; en retour, les environnements sociaux et physiques ainsi modifiés exercent des déterminations qui conditionnent et affectent la poursuite des activités initiales. De plus, à toutes les étapes de ces boucles de rétroaction jouent des relations, en boucles également, par lesquelles certaines actions individuelles produisent un effet social (d'où le concept d'amplification socioficatrice) et des états de choses collectifs produisent des effets sur les comportements individuels. 
PHILOSOPHIQUeS, Vol. XVI, Numéro 1, Printemps 1989

\title{
UN MODÈLE GÉNÉRAL \\ POUR DÉCOMPOSER LA RELATION D'IMPACT DANS LES RECHERCHES SUR L'IMPACT SOCIAL DE LA SCIENCE ET DE LA TECHNOLOGIE
}

\author{
par Normand Lacharité
}

\begin{abstract}
RÉSUMÉ. L'impact social de la science et de la technologie se manifeste par une série de relations que le présent modèle va découper sur des boucles de rétroaction. L'activité techno-scientifique des humains crée des perturbations dans le réseau des relations interindividuelles et sociales ainsi que dans l'environnement physique; en retour, les environnements sociaux et physiques ainsi modifiés exercent des déterminations qui conditionnent et affectent la poursuite des activités initiales. De plus, à toutes les étapes de ces boucles de rétroaction jouent des relations, en boucles également, par lesquelles certaines actions individuelles produisent un effet social (d'où le concept d'amplification socioficatrice) et des états de choses collectifs produisent des effets sur les comportements individuels.
\end{abstract}

ABSTRACT. Science and technology affect social life through numerous relations and the present model undertakes to picture the main ones as specific portions of feedback cycles. By its very creativity in science and technology man induces changes in the network of interindividual and social relations, and drastically modifies the physical environment ; those social and physical changes, in turn, serve as conditions and causes for retroactive changes in the human scientific and technological behavior. Furthermore, the model introduces the concept of «amplification socioficatrice» (this neologism might be rendered by "sociogenic amplification") in order to highlight those additional relations through which certain individual actions or reactions come to have a social effect and existence in the midst of the generalized loop relations obtaining between individual(s) and society. 
Plan de l'essai

I. (Introduction) EXPLICATIONS TERMINOLOGIQUES

II. ESQUISSE DU MODĖLE

1. L'interface idéation/mouvement

2. La production émanant de $\mathrm{SI}_{\mathrm{n}}$; ou : l'inscription des extrants de $S I_{n}$ dans son environnement

3. L'amplification socioficatrice des produits émanant d'agents SI,

4. L'environnementalisation des produits de l'action

5. L'action en retour de l'environnement sur les systèmes d'action individuels

5.1 La saisie d'information

5.2 L'utilisation d'artefacts techniques

5.3 L'environnement externe $\mathrm{E}_{\mathrm{x}}$ comme terme-source de relations causales naturelles

5.3.1 La distinction entre activité techno-scientifique et activités d'une autre sorte

5.3.2 Le problème de l'existence même d'effets repérables sur des systèmes individuels

5.3.3 Les lois de la nature et l'autonomie des systèmes individuels

6. L'amplification socioficatrice des réactions individuelles à l'environnement techno-modifié

III. (Conclusion) QUALITÉS, LIMITES ET LACUNES DU PRÉSENT MODÈLE

EXPLICATIONS TERMINOLOGIQUES

(Introduction)

Le modèle esquissé ici appartient au groupe de ce que l'on appelle des modèles d'impact. Il s'agit d'un modèle à cycles, à deux niveaux de complexité.

Par modèles à cycles, j'entends des modèles qui représentent les chaînes causales comme des boucles de rétroaction, ou fragments de telles boucles. On peut expliciter cette caractéristique en disant que ces modèles satisfont ou acceptent tous le postulat de l'impact rétroactif, c'est-à-dire la proposition suivante:

Toute cause est potentiellement affectée, au terme d'un délai plus ou moins long, et de relais plus ou moins nombreux, par l'action sur elle que peut excrcer en retour son effet ou une partie de son effet ou encore une combinaison de ses effets. 
Quant aux deux niveaux de complexité du modèle ce sont, grosso modo, ceux qui déterminent respectivement les systèmes définis sur les individus humains ou personnes et ceux définis sur des groupes d'individus.

Dans ce qui suit, j'utiliserai fréquemment l'expression les individus bumains pour abréger l'expression plus correcte mais plus lourde les systèmes définis sur les individus humains. Même chose pour l'expression les groupes sociaux utilisée pour abréger: les systèmes définis sur les groupes sociaux. Les formulations plus lourdes servent à marquer, lorsque c'est utile, le caractère construit des concepts utilisés dans la théorie pour parler des entités du monde concret (en particulier le fait que ces concepts dépendent de définitions et postulats marqués par l'option systémique).

\section{II \\ ESQUISSE DU MODÈLE}

On pose d'abord qu'un certain nombre de systèmes individuels $\mathrm{SI}_{\mathrm{i}}(\mathrm{i}=1,2,3, \ldots)$ sont reliés entre eux de façon à constituer toutes les composantes, et elles seules, d'un système social SS. (Le symbole $S S$ est considéré ici comme une constante).

\section{L'interface idéation-mouvement}

En principe, toutes les relations que je m'apprête à décrire font partie d'une ou plusieurs boucles de rétroaction; c'est par une décision arbitraire que je choisis sur la boucle la relation que je décrirai en premier lieu. Convenons que cette relation est la relation d'action qui, à un acte d'idéation attribué à un système individuel particulier, fait correspondre un comportement observable de ce système.

Pour donner un exemple typique : le système individuel $\mathrm{SI}_{\mathrm{n}}$ (n appartient à $\mathrm{I}$; et I est l'ensemble des indices d'individuation $\mathrm{i}=1,2,3, \ldots)$ conçoit une action et la réalise. La réalisation d'une action s'entend ici comme la mise en œuvre de l'un ou l'autre des organes moteurs de $\mathrm{SI}_{n}$ par lesquels $\mathrm{SI}_{n}$ transmet de l'énergie et/ou de l'information à des objets ou états de choses situés dans son environnement. 
Dans la présente conceptualité, toutes les «nouveautés» scientifiques et techniques, que ce soit des propositions et représentations ajoutées au corpus des savoirs, ou des procédures, ou des objets-ustensiles ou des objets-machines, sont réputées avoir une origine relative (pas nécessairement une origine absolue, au sens d'origine à partir de rien) dans un processus idéationnel interne à $\mathrm{SI}_{n}$ et subséquemment manifesté par des mouvements de $\mathrm{SI}_{\mathrm{n}}$ moyennant une transmission réussie d'information appropriée, à l'interface entre les organes de l'idéation et ceux qui réalisent des mouvements.

\section{La production émanant de $S I_{n}$; ou : l'inscription des extrants de $\mathrm{SI}_{\mathrm{n}}$ dans son environnement}

La production imputable à $\mathrm{SI}_{n}$, et consécutive à l'action de $\mathrm{SI}_{\mathrm{n}}$, est considérée ici comme inscription, dans l'environnement, des transformations dues aux mouvements de $\mathrm{SI}_{\mathrm{n}}$. Une façon de décrire cette inscription, façon primitive sans doute mais non moins fondamentale, est de relever les changements d'états des objets ou états de choses qui sont reliés par des relations causales aux mouvements de $\mathrm{SI}_{n}$. Naturellement, l'inventaire de ces relations causales est affaire de théorisation et de modélisation et dépassera normalement de beaucoup le niveau de l'observation naïve qui prétendrait les trouver toutes déjà données dans une description purement behavioriste, ou se voulant telle, du comportement de $\mathrm{SI}_{\mathrm{n}}$; le problème de trouver les termes pertinents de toutes les relations causales dans lesquelles un événement quelconque est impliqué, et à plus forte raison un système actif intentionnel comme $\mathrm{SI}_{n}$, est ni plus, ni moins central ici que dans n'importe quelle investigation scientifique, et n'est pas plus propre à l'interface entre $\mathrm{SI}_{n}$ et son environnement qu'à n'importe quelle autre transition survenant sur le parcours des boucles de rétroaction que je décris ici.

Pour évoquer des formes un peu plus concrètes de la production imputable à $\mathrm{SI}_{n}$, on peut expliciter les types de production suivants :

- les artefacts langagiers, qui sont des fragments de discours manifestés par des fragments du langage parlé ou écrit ; le 
terme inscription, dans une acception un peu plus spécifique que celle utilisée ci-dessus, désigne parfois les artefacts langagiers en tant que fixés sur un support matériel qui en rend l'occurrence reproductible (par exemple, lorsqu'un disque ou une bande magnétique a fixé des fragments de langage parlé) ou qui du moins transforme l'occurrence en état de choses, rendant ainsi possible un nombre quelconque, souvent très grand, d'actes de lecture à l'égard desquels le fragment langagier fixé devient l'équivalent d'une réoccurrence du fragment initial (par exemple, lorsqu'un fragment est gravé sur une pierre tombale ou imprimé dans un livre).

- les artefacts techniques, tels les objets manufacturés, les maisons, les outils, les machines, etc., de même que les procédures ou procédés impliqués dans la production ou l'usage des items précédents et, d'une façon générale, impliqués dans la performance de chaînes relativement stéréotypées et codifiées de gestes. Ici la procédure utilisée pour extraire du pétrole des sables bitumineux satisfait la définition que je donne d'un artefact technique au même titre que la procédure au sens juridique et que la procédure pour chasser les démons, réussir le koulibiac ou traiter la vérole.

- les perturbations écophysiques, qui sont des changements d'état du milieu physique, changements considérés comme l'inscription des extrants de $\mathrm{SI}_{\mathrm{n}}$ ou, pour le dire plus simplement, comme résultats des actions de $\mathrm{SI}_{\mathrm{n}}$. (Le terme perturbation n'a pas ici de valeur péjorative ; entendons-le comme en mécanique théorique). Naturellement, on peut définir pour $\mathrm{SI}_{n}$ un milieu, disons, émotionnel et considérer que des mouvements de $\mathrm{SI}_{n}$ ont valeur d'actions qui provoquent des changements dans ce milieu; et ainsi de suite, pour les divers types de milieux que la conceptualité théorique permet ou exige de distinguer. 


\section{L'amplification socioficatrice des produits émanant d'agents $\mathrm{SI}_{\mathrm{i}}$}

Dans tout modèle qui " part » en décrivant des actions imputables à un système individuel et qui vise à décrire des phénomènes sociaux, doivent se trouver explicités quelque part les processus par lesquels certaines des activités d'agents individuels ont des produits qui s'inscrivent dans l'environnement social ou, si l'on veut, qui constituent, en le transformant, l'environnement spécifiquement social. Le caractère social de certains produits d'activités individuelles s'acquiert; et il ne s'acquiert que relativement à certaines activités (non pas toutes) et à certains produits (non pas tous); il ne s'acquiert donc aussi que relativement à certains individus, et non pas tous. Dire que des activités par leurs produits, acquièrent un caractère social, c'est dire tout aussi bien que le social advient; les deux formulations désignent une seule et même réalité ; et c'est cet avènement du social que je veux signaler par le néologisme «sociofication » : les processus que je vais partiellement décrire sont socioficateurs en ce sens que c'est par eux (toujours selon les postulats du présent modèle, et seulement dans les limites de leur applicabilité) que la société «se fait».

L'idée générale qui guide ma conception des processus par lesquels un produit d'action devient social, je la nomme amplification socioficatrice et je l'explicite sous la forme suivante. Je considère d'abord ce produit d'action, conformément à la conceptualité systémique, comme un terme possible de relations d'action dans lesquelles il va figurer comme terme-source plutôt que comme terme-cible (en reliant le produit à l'action d'un agent, je l'ai jusqu'à présent considéré seulement comme terme-cible, de la manière suivante :

$$
\text { agent } \longrightarrow \text { produit }
$$

la flèche représentant la relation d'action). L'amplification socioficatrice consiste en ce qu'un produit quelconque devienne le termesource d'un nombre élevé (je commenterai cet adjectif tout à l'heure) de relations d'action ayant comme terme-cible des agents quelconques, parmi lesquels figurent nécessairement (et surtout) des agents autres que l'auteur initial du produit concerné. Cette multiplication des relations d'action dont le produit est la source correspond à l'amplitude de l'influence exercée par le produit 
concerné sur autrui ; cette multiplication est conçue ici exclusivement en termes numériques et la fonction qui la définirait dans une version formelle du modèle serait un scalaire.

Plusieurs situations ou processus peuvent réaliser l'accroissement du nombre des relations d'action dont il s'agit ; sans prétendre les énumérer exhaustivement je mentionne les suivants:

— le produit est lui-même «reproduit »à un nombre élevé d'exemplaires. Soit qu'un même agent est responsable de la reproduction (il possède, par exemple des moyens de reproduction; la technologie, à un certain stade de son développement, a consisté presqu'entièrement à institutionnaliser dans des machines et des rapports de travail la reproduction d'un produit à un grand nombre d'exemplaires), soit que plusieurs agents accomplissent une certaine activité dont les produits sont des exemplaires les uns des autres.

- le produit, sans être à proprement parler reproduit, est pris en charge par un « diffuseur " qui établit de nombreuses relations d'action entre ce produit et des termes-cibles. Le paradigme des processus de ce genre est sans doute le cas où le produit est principalement un contenu informationnel (un discours parlé, un spectacle audio-visuel) et est pris en charge par un médium d'information (radio, télé, par exemple).

- le produit, en s'inscrivant dans l'univers physique, engendre une ou des transformations qui, pour un nombre élevé de systèmes individuels ayant un environnement défini sur cet univers physique, ont valeur de perturbations capables de générer des réactions chez les systèmes $\mathrm{SI}_{\mathrm{i}}$. Il n’importe pas ici que la réaction soit consciente, il importe seulement que les relations entre la perturbation (comme changement d'état causé par l'inscription du produit dans l'environnement $\mathrm{E}$ ) et la réaction (comme changement d'état d'un $\mathrm{SI}_{n}$ cible, dans l'environnement $\mathrm{E}$ ) soient assez nombreuses pour que leur coexistence forme un état de choses collectif (ou état d'une collectivité de systèmes individuels). On doit remarquer ici la distinction que je fais entre le concept d'univers physique, qui est le concept nécessaire à 
la définition d'un extrant d'un système individuel, et le concept d'environnement pour des systèmes individuels : la perturbation et les réactions qu'elle entraîne ne sont pas nécessairement physiques, elles peuvent être esthétiques, psychologiques, émotionnelles, spirituelles, etc., mais tous les environnements susceptibles d'être spécifiés par ces adjectifs sont définis sur l'univers physique et toute perturbation survenant dans l'un de ces environnements est toujours le fait d'un produit d'action qui «s'inscrit » dans l'univers physique. (L'univers physique est un support générique obligé, quels que soient les processus envisagés, en vertu d'un postulat ontologique matérialiste que j'entends faire ici valoir pour tous les systèmes considérés). Ce que je décris ici pourrait peut-être s'appeler processus d'atteinte àl'environnement collectif; et la manière dont ce processus donne lieu à une amplification socioficatrice est énoncée dans les deux relations suivantes:

$1^{\circ}$ plus un produit atteint en profondeur, via son inscription physique, la configuration de l'environnement commun à un nombre donné de systèmes individuels, plus il a de chances d'exercer une influence sur les systèmes individuels partageant cet environnement.

$2^{\circ}$ définissons l'importance socio-extensionnelle d'un environnement comme une propriété qui crôit proportionnellement au nombre de systèmes individuels $\mathrm{SI}_{\mathrm{i}}$ qui partagent cet environnement; alors, plus est grande l'importance socio-extensionnelle de l'environnement qu'atteint, à un degré de profondeur donné, un produit d'action, via son inscription dans l'univers physique, plus grand est le nombre des relations probables d'influence dont ce produit est un terme-source. Par cette nouvelle relation de proportionnalité, un produit peut augmenter encore ses chances d'exercer une influence sur des systèmes individuels.

Maintenant la question qui reste en suspens est la suivante : combien élevé doit être le nombre des relations d'influence qui relient un produit d'action à des systèmes individuels pour qu'on puisse qualifier de social l'effet généré par le produit concerné ? La 
réponse paraît simple, si on la formule de la façon suivante : ce nombre doit être du même ordre de grandeur que le nombre des systèmes individuels qui composent les collectifs sur lesquels un observateur donné (un chercheur en sciences humaines, par exemple) est prêt à définir des processus sociaux. Mais la simplicité de cette réponse n'est qu'apparente, car elle renvoie le problème à la détermination des critères par lesquels, dans chaque contexte de recherche ou d'observation, se trouve délimité le système d'action (pour employer la terminologie de Parsons et des systémistes) pertinent, eu égard aux problèmes posés et aux objectifs poursuivis. Il est bien qu'il en soit ainsi, car il n'y a pas de critère numérique du social; il n'y a jamais que des jeux de relations définis sur des regroupements de personnes; et les systèmes individuels dont je parle ne sont eux-mêmes que des jeux de relations (donc des constructions conceptuelles) définis sur des personnes.

La sociofication que je tente de décrire ici peut en grande partie se décrire aussi bien comme la réaction de certains « systèmes d'action» à l'action que des produits suffisamment nombreux exercent sur l'environnement de ces mêmes systèmes d'action. Cette façon de dire soulève pour moi deux problèmes importants que je voudrais maintenant évoquer rapidement; le premier problème s'énonce : quel est le support ontologique de la capacité d'agir imputée aux systèmes d'action? le deuxième problème s'énonce : quelles sont les relations d'extériorité appropriées à la description des rapports entre des systèmes d'action et leurs divers environnements, étant admis que certains systèmes d'action peuvent constituer des environnements pour certains autres? Je voudrais, avant de passer à la section 4 consacrée à « l'environnementalisation des produits de l'action », indiquer une ou deux idées concernant la manière dont ces problèmes se posent.

Le premier problème, celui de l'ontologie du mode d'action propre aux systèmes d'action, est l'un de ceux que l'on discute encore souvent dans les sciences sociales, à l'occasion du débat entre individualisme et holisme. On demande, par exemple : est-ce que la société est un véritable "agent», c'est-à-dire un système dont on puisse dire qu'il est actif en présupposant les mêmes postulats ontologiques que ceux qu'on admet lorsqu'on dit d'une personne qu'elle constitue un système « actif ». Il n'est pas nécessaire 
de présupposer ici que les groupes sociaux sont des systèmes actifs ou qu'ils ne le sont pas ; mais, bien sûr, selon les présupposés qu'on adoptera, le nombre et la variété des relations causales qu'on admettra dans le modèle vont fluctuer. Pour ma part, je considère les groupes sociaux comme des systèmes non actifs, mais je n'en fais pas une thèse, car je ne saurais la démontrer; une telle démonstration exigerait d'abord une théorie qui fournisse les conditions nécessaires et suffisantes pour qu'un système soit à bon droit caractérisé comme actif au sens de "doté d'une source autonome d'énergie de réaction", et je ne connais pas de telle théorie ; certes, la clôture organisationnelle apparaît comme l'une des plus explicatives des conditions nécessaires de l'autonomie des systèmes vivants, conformément à ce qu'en affirment Varela et Maturana; mais cette circonstance est de peu de secours, si j'observe :

$1^{\circ}$ que mon concept de système actif n'implique pas le concept de système vivant (pour être actif, au sens visé, un système n'a pas nécessairement à être vivant, il me semble);

$2^{\circ}$ qu'un groupe social quelconque ne manisfeste pas toujours et en général une clôture organisationnelle, mais seulement à l'occasion et seulement par certaines composantes de son processus complexe de reproduction;

$3^{\circ}$ que la question de savoir si l'autonomie du vivant (en tant que propriété systémique) s'applique à un système du genre groupe social dépend de deux questions subsidiaires auxquelles je ne connais pas de solution générale :

Q1 dans quels cas un système possède-t-il une propriété générale partagée par ses composantes (un système composé de cellules a-t-il un fonctionnement cellulaire ? partiellement ou occasionnellement cellulaire ?) ? Plus spécifiquement et plus suggestivement : un système composé de systèmes autonomes est-il de ce fait autonome?

Q2 l'autonomie du vivant s'applique-t-elle à l'individu humain considéré comme système intentionnel ou cesse-t-elle déjà là de s'appliquer? Et si un système 
intentionnel de ce genre est autonome, est-ce encore au sens que la théorie de l'autopoièse tente d'expliciter? ( $\grave{A}$ moins d'affaiblir considérablement le concept d'autopoièse, il m'apparaît déjà fort invraisemblable qu'un système intentionnel soit autopoiétique, à cause du caractère foncièrement aléatoire et erratique de ses représentations).

Naturellement, un certain nombre de problèmes ontologiques relatifs au statut et à l'identité de l'agent peuvent être esquivés si l'on s'en tient au concept plus général, et plus métaphysique, de cause. Au sens le plus large, une cause est une source de déterminations pour autre chose. Un système quelconque n'a pas besoin d'être un système actif pour être une cause. De même, un objet, état de choses ou événement n'a pas besoin d'être un système pour être une cause, c'est-à-dire pour exercer des déterminations ou perturbations. Mais là encore : que recèle exercer?

Je poursuivrai quelque peu les remarques précédentes dans l'introduction de la section 5.3 ci-dessous.

Le deuxième problème que j'avais annoncé est celui de la spécification des relations d'extériorité, en présence de systèmes d'action plus ou moins complexes. Durant la phase de l'amplification socioficatrice, l'action que nous cherchons à décrire passe d'un niveau de complexité donné à un niveau de complexité supérieur dans le sens suivant : alors que les processus survenant à l'interface idéation-mouvement peuvent généralement être décrits en majeure partie sur un système ayant la complexité du système individuel, les processus d'amplification socioficatrice ne peuvent être décrits, en ce qu'ils ont de spécifique (je ne parle même pas encore d'une description adéquate et exhaustive), que sur un système de complexité supérieure, caractérisé en première approximation par le fait, justement, qu'il comporte, à titre de sous-systèmes, les systèmes individuels précédemment mentionnés. Or une difficulté conceptuelle propre à ce genre de « passage » - et pour moi, elle est présentement la plus centrale par ses enjeux - est de définir clairement la différence entre l'environnement par rapport auquel penser le système d'action de niveau social et l'environnement par rapport auquel penser le système d'action de niveau individuel. De quelque façon, si j'appartiens, avec d'autres systèmes individuels, à un 
système social donné, disons $\mathrm{S}_{\mathrm{m}}$, ces systèmes individuels qui sont mon autrui ne me sont pas «extérieurs 》 au même titre que les montagnes ou les rues, les livres ou les spectacles, les autres groupes sociaux, etc., desquels nous pouvons dire qu' ils forment ou occupent notre environnement commun et qu'ils sont, à ce titre, extérieurs à nous tous en tant que sociétaires de $S_{m}$.

Il y a des degrés d'extériorité, pour ainsi dire; prenant une analogie, on peut dire que le système respiratoire $\mathrm{R}$ d'un être vivant $S_{v}$ est «moins extérieur » au système circulatoire $C$ du même $S_{v}$, que les systèmes routiers ou écologiques dans lesquels se meut $S_{v}$; R entretient cependant des relations réelles, et directes, aussi bien avec $C$, dans $S_{v}$, qu'avec l'environnement de $S_{v}$. Et, dans ce cas des organismes vivants, on possède un fait empirique bien avéré qui vient donner appui à l'intuition selon laquelle tous les systèmes-compléments de $\mathrm{R}$ sur $\mathrm{S}_{\mathrm{v}}$ sont moins extérieurs à $\mathrm{R}$ que ne l'est l'environnement écologique: c'est le fait que toutes les cellules de $\mathrm{R}$ et $\mathrm{C}$ possèdent le code génétique propre à $\mathrm{S}_{\mathrm{v}}$ comme individu organique ; l'état de choses le plus déterminant, peut-être, de l'unité de $S_{v}$ comme système, à savoir le code génétique, est présent dans $\mathrm{R}$ lui-même à la fois comme facteur d'unité fonctionnelle de $\mathrm{R}$ et comme critère de son identité numérique (au sens suivant: $\mathrm{R}$ est le système respiratoire de Paul et non celui de Jacques). C'est cette intériorisation par $\mathrm{R}$ d'une propriété essentielle de $S_{v}$ qui oblige à penser que la relation de $R$ à $S_{v}$ n'est pas autant une relation d'extériorité (si tant est qu'elle en est une) que la relation de $\mathrm{R}$ à l'environnement écologique. La distinction concernée est parfois conceptualisée en différenciant environnement interne et environnement externe; je ne sais si cette différenciation suffit - je soupçonne que non; une différenciation par degrés me semble requise si on veut une conceptualité générale -, mais je la crois nécessaire et pertinente, lorsqu'il s'agit de penser la manière dont les individus composant un socio-système intériorisent des traits de ce socio-système au titre même de composantes de leur identité (il s'agit de leur identité spécifique et non de leur identité numérique; par exemple : identité comme Québécois, plutôt que identité comme Jacques), lorsqu'il s'agit, par conséquent, de penser la relation des individus à des socio-systèmes conçus comme environnements; car même ainsi conçus, ces socio-systèmes ne sont pas des environnements au sens plus «extérieur» dont il s'agira dans la section suivante. 
À l'égard de la problématique précédente et des exemples au moyen desquels je l'illustre, je crois utile de faire la précaution oratoire suivante : je n'endosse pas, au cours de ces considérations, le point de vue qu'on a souvent appelé organiciste à propos de la société. Je sais que les métaphores organicistes ne sont pas prisées en théories contemporaines de la société et cette méfiance est certainement justifiée lorsque les métaphores concernées finissent par impliquer l'idée que c'est la nature qui détermine les rapports sociaux (comme elle le fait pour les rapports entre les organes) et que les rapports hiérarchiques, orientés vers une fin «naturelle», marqués par la collaboration, constituent un modèle que la nature propose à notre action instauratrice ou impose peut-être même, sous la forme d'une harmonie pré-établie cachée sous les apparences des conflits agitant constamment les humains. Cependant, je maintiens que la comparaison avec les sous-systèmes des êtres vivants est ici féconde et légitime (plutôt que "misleading» et idéologiquement biaisée) précisément pour ce qui est de penser la "logique» du concept d'environnement et des relations entre système et environnement. En parlant des systèmes vivants on peut admettre, je pense, la thèse suivante: Le génotype d'un organisme multicellulaire n'est pas extérieur à cet organisme; et cependant, au sein d'un génotype donné, un individu est dans une relation d'extériorité par rapport à un autre individu. Au niveau du phénotype, la thèse pourrait être reprise de la manière suivante: Le code génétique d'un individu pluricellulaire n'est pas extérieur à une cellule de cet individu et cependant, au sein d'un phénotype donné, une cellule est dans une relation d'extériorité par rapport à une autre cellule. Je crois que ces exemples sont pertinents, lorsqu'il s'agit de réfléchir à la logique de la relation d'extériorité dans le contexte où cette relation doit composer avec la relation système/sous-système.

\section{L'environnementalisation des produits de l'action}

Le néologisme environnementalisation veut exprimer l'idée du processus par lequel quelque chose qui n'était pas une composante de l'environnement d'un système d'action devient une composante de cet environnement. 
À l'issue des phases précédemment décrites du modèle en cours de construction, nous sommes en présence d'un sociosystème $S_{s}$ dont l'action est capable de produire tous les artefacts que nous voudrons bien faire entrer dans la définition (du moins ostensive et nominale) de science et technologie. Par une simplification méthodologique inspirée des propos précédents concernant les relations d'extériorité, je conviens de définir un environnement externe $\mathrm{E}_{\mathrm{x}}$ comme étant l'environnement partagé en commun par tous les sous-systèmes individuels de $S_{s}$ sans qu'aucun des soussystèmes individuels n'appartienne lui-même à cet environnement, du moins tant et aussi longtemps que chacun est considéré comme un système d'action. L'objectif de cette simplification méthodologique est de proposer un concept d'environnement qui s'applique exclusivement à la conjonction des composantes du monde naturel et des artefacts résultant de l'activité techno-scientifique des systèmes d'action individuels ou collectifs. Je ne prétends pas que ce concept soit satisfaisant en tous points; mais je fais l'hypothèse qu'il faut le construire, lui ou un semblable, pour le distinguer des concepts applicables aux environnements moins externes et plus abstraits qui conviennent à la description des activités politiques, économiques, culturelles, etc., des mêmes systèmes d'action.

Le modèle en cours d'élaboration comporte la présente phase d'environnementalisation pour permettre la description explicite, et pour elle-même, des transformations qui résultent de l'inscription dans l'univers physique des produits de l'activité techno-scientifique imputable aux systèmes d'action, compte tenu de tous les processus subsumés déjà par moi sous le concept d'amplification socioficatrice. Beaucoup de descriptions de l'impact de l'activité techno-scientifique se font en termes de ce qui disparaît de l'environnement externe ou de ce qui y apparaît ; typiquement, ce qui disparaît, ce sont, par exemple, des ressources naturelles non renouvelables; et ce qui y apparaît, ce sont, par exemple, des machines, des habitations, des villes.

À cette étape du modèle, il convient de circonscrire quels sont les produits qui acquièrent le statut de composantes de l'environnement $\mathrm{E}_{\mathrm{x}}$ par opposition à ceux qui ne l'acquièrent pas (parce qu'ils ne sont, disons, pas assez durables); de chercher les conditions auxquelles la sédimentation ou accumulation des produits est possible. Ces deux problèmes se posent d'une façon particulièrement 
alambiquée, lorsque les produits considérés valent surtout comme information et peuvent être considérés comme tels. D'abord l'information a des rapports très compliqués à l'espace et au temps, du fait qu'elle n'est jamais qu'accessoirement (ou accidentellement) caractérisée par les propriétés physiques de ses porteurs et que ses paramètres d'identification sont à puiser dans des classes d'occurrences possibles (donc dans des espaces logiques), dans les propriétés logiques des stocks de signes pertinents et dans les réseaux de relations (entre états des systèmes individuels) qui constituent la convention nécessaire au décodage. Dans ces conditions, il est difficile de définir le type d'extériorité qui permette de reconnaître une information comme composante (disponible) de l'espace $\mathrm{E}_{\mathrm{x}}$ commun aux systèmes d'action, et ce, sans qu'il faille supposer pour autant qu'elle ait quitté le système qui l'a produite (et qu'elle n'y soit par conséquent plus présente) ; un système perd l'énergie ou la matière qu'il délivre en sortie, selon des comptabilités prévues par les lois de conservation formulées en physique, mais il n'y a pas de telles lois pour régir les flux d'information échangés entre systèmes. Ces particularités ontologiques des processus et produits valant comme information rendent également compliqué le traitement du second problème, celui de la sédimentationaccumulation, car il n'y a pas, à ma connaissance, de modèle un tant soit peu général de la manière dont se comporte dans la durée, à son niveau d'invariance temporelle propre, un produit informationnel externalisé; ni de modèle de la manière dont de tels produits provenant de sources différentes peuvent s'agglutiner ou se neutraliser, ou nouer entre eux quelqu'autre relation. Il ne s'agit pas d'imaginer, ici, des lois d'évolution pour des produits informationnels qui seraient considérés comme des systèmes actifs (dotés d'un principe autonome d'action dont ils sont le siège et l'origine); ils ne sont pas des systèmes actifs, ils ne disposent même pas des principes internes d'activité propres aux systèmes inanimés que sont les astres et les atomes; le capital ne s'accumule pas tout seul, l'information non plus; tous les deux sont des entités passives, objects possibles d'attitudes ou actions humaines. Cependant, même après avoir limité par ces précautions la conception d'un "environnement informationnel», il s'agit tout de même de produire le concept d'une accumulation d'information ayant le statut de condition objective externe et préalable, relativement à l'activité des systèmes (humains) d'action à l'égard desquels ces 
informations sont justement déclarées disponibles avec et en vertu d'un agencement aussi réel qu'arbitraire. Et, dans la mesure où l'on veut bien considérer les connaissances comme un type particulier d'environnement informationnel, c'est justement là la tâche d'une écologie des connaissances.

\section{L'action en retour de l'environnement sur les systèmes d'action individuels}

Tout état de l'environnement $\mathrm{E}_{\mathrm{x}}$ est une source virtuelle de déterminations, et donc d'effets, pour les systèmes d'action en général, et ce, en tant que conditions objectives réalisées capables d'exercer des contraintes, à la fois comme conditions de possibilité et comme conditions circonstancielles affectant le déroulement du comportement des systèmes d'action.

Dans l'énoncé précédent, les conditions sont dites exercerdes contraintes ; il existe au moins deux autres manières de déterminer des systèmes d'action, dans ce contexte:

$1^{\circ}$ créer des perturbations; auquel cas, on dit que les systèmes d'action réagissent; l'adaptation, l'accommodation, le "feedback. positif ou négatif, etc., sont des types de réaction.

$2^{\circ}$ offrir des possibilités; dans ce cas, on dit que les systèmes saisissent (ou non) ces possibilités, qu'ils les mettent à profit (ou non), etc.

Le modèle d'impact que je propose ici admet l'un ou l'autre des trois langages mentionnés: celui des contraintes, celui des perturbations, et celui des possibilités.

Comme pour les portions du modèle consacrées à décrire des relations allant des systèmes d'action vers l'environnement, je distingue, au moment de décrire les relations inverses, le palier des effers définissables sur les systèmes individuels et le palier des effets définissables sur les systèmes collectifs. (Je traiterai de ces derniers dans la section 6.) Cette option théorique, qui a des conséquences méthodologiques considérables, tant pour la cueillette des données que pour leur généralisation, explication et modélisation, est exigée logiquement par le postulat déjà adopté ci-dessus, 
stipulant que seuls des systèmes individuels peuvent être actifs au sens fort de: "possédant en eux-mêmes un principe autonome d'action».

Je vais distinguer trois types de processus par lesquels des éléments de $\mathrm{E}_{\mathrm{x}}$ (objets, états de choses, événements) peuvent avoir un impact sur des systèmes individuels (bien entendu, dans son état présent, cette typologie n'a malheureusement rien de systématique; elle n'est certainement pas objective; elle n'est peut-être même pas homogène) :

- la saisie d'information;

- l'utilisation d'artefacts techniques;

— l'«action » de $\mathrm{E}_{\mathrm{x}}$ considéré comme terme-source de relations causales «naturelles».

Les deux premiers types nomment des actions dont l'initiative (ce concept aussi présente des difficultés de définition) appartient à un système individuel humain: saisir, utiliser; le dernier type nomme une action dont l'initiative appartient à la nature.

\subsection{La saisie d'information}

Les produits informationnels inscrits matériellement, c'est$\grave{a}$-dire physiquement, dans l'evironnement $E_{x}$ défini ci-dessus constituent autant de contraintes ou de possibilités, relativement aux comportements éventuels des systèmes d'action individuels; et si l'on considère un changement d'état dans la masse des produits informationnels activables (activable: susceptible d'être interprété par un système individuel), alors cette occurrence constitue une perturbation du milieu, relativement aux comportements éventuels des mêmes systèmes.

Des systèmes interprétants, généralement différents des producteurs initiaux, peuvent saisir l'information disponible dans $\mathrm{E}_{\mathrm{x}}$, et s'ils le font, un flux d'information passe de $E_{x}$ vers le système interprétant, et réintègre par cette médiation (comme par une porte d'entrée) le système d'action $\mathrm{S}_{s}$. Ce processus est un processus cognitif, en ce sens qu'il amène le flux d'information aux organes de l'idéation, dans un système individuel donné. Et dans la mesure où le cheminement que j'ai décrit jusqu'à présent commençait avec l'idéation, le modèle boucle ici un cycle. Ce cycle constitue une 
partie intéressante du modèle d'impact en ce qu'il tente de caractériser pour lui-même le réseau des chemins qu'emprunte la composante informationnelle des diverses relations d'action; c'est à cette composante qu'appartient la denrée particulière qui s'appelle les connaissances scientifiques et techniques. Bien que le système des unités selon lesquelles il convient d'individuer, de compter et de mesurer des morceaux de cette denrée soit encore très rudimentaire (pensons aux travaux de «scientométrie», illustrés par ceux du pionnier Derek de Solla Price), un bon modèle d'impact doit prendre acte des diverses propriétés que cette denrée assume dans la suite contingente de ses modes d'apparition et d'existence : non seulement les chemins qu'elle parcourt, mais aussi les points d'accumulation, de naissance ou d'extinction ; les modes de transmission ; les conventions, présupposés, valeurs, exclusions, émotions, etc. Or ces propriétés ne sont décelables et correctement descriptibles, pour la plupart, que dans le processus par lequel un système individuel, puis plusieurs, saisit l'information passive, latente, disponible, permettant ainsi de déterminer où le flux d'information se dirige, et quel est son contenu.

Le traitement de ces questions relève aussi d'une écologie des connaissances; et celle-ci, pour ce qui est du présent processus, trouvera sans doute une partie importante de ses cadres théoriques et de ses données dans la sociologie des connaissances, mais devra sans doute aussi les renouveler considérablement pour les rendre compatibles avec l'approche informationnelle systémique.

Finalement, à la présente étape du modèle, doivent également être étudiés les événements qu'on pourrait appeler événements négatifs, ici réalisés par les cas où l'information, bien que matériellement disponible et techniquement accessible, n'est pas réinsérée dans des systèmes d'actions individuels et reste « morte ». Plusieurs phénomènes sont susceptibles d'intérêt à titre d'événements négatifs. Je pense, en premier lieu, aux cas où l'information cesse de circuler du fait de devenir caduque; dans le contexte d'un rapport à des utilisateurs potentiels, il y aurait peut-être moyen de définir un concept cohérent de taux d'obsolescence pour des types d'information et des discours, tout comme pour des outils techniques. Il y a ensuite des manques à circuler dus à des forces d'exclusion, avec ou sans intervention consciente d'agents individuels; Foucault, dans L'Ordre du discours, faisait volontiers l'hypothèse que certains 
discours sont exclus de la scène des échanges discursifs entre les humains, du simple fait que certains discours occupent déjà la place ; les idées (peut-être pas toujours claires) de discours dominant et d'idéologie dominante ont beaucoup servi à développer ce thème. À son tour, l'idée qu'un discours peut en quelque sorte saturer l'espace discursif (et de ce fait exclure d'autres discours) évoque le thème des capacités limites des canaux de communication ; ce thème a été parmi les tout premiers à être étudiés en théorie de l'information par les mathématiciens et les ingénieurs et se retrouve maintenant au cœur de la théorie de la recherche: la prolifération de l'information scientifique et technique donne lieu à divers phénomènes d'engorgement ( overloading ») des canaux de communication avec lesquels un individu parvient à fonctionner. Il est certain que l'aisance avec laquelle les canaux de communication de type électronique ont sans cesse augmenté leur puissance de transmission n'a guère son équivalent dans les structures psychologiques et physiologiques des systèmes d'action individuels. Je ne serais pas surpris qu'un type particulier de résistance aux flux d'information soit engendré par le fait de la démesure que prend la supériorité des médias artificiels de transmission d'information, en nombre et en puissance, sur les médias bio-psychologiques et sociologiques. Et s'il en est ainsi, il y a une sorte particulière de "déchets» ou «rejets» d'information qui tient à la faiblesse du récepteur humain en regard des sources qui, une fois sollicitées, le bombardent; ces déchets ralentissent-ils la circulation de l'information pertinente? s'accumulent-ils -- et, le cas échéant : où? dans la réalité que tente de décrire le présent modèle d'impact?

\subsection{L'utilisation d'artefacts techniques}

Lorsqu'ils sont utilisés par des systèmes d'action individuels, les artefacts techniques sont intégrés à un complexe de chaînes causales, principalement intentionnelles (je veux dire par là que la relation causale mécaniste y joue une part infime et asservie, et que toutes les relations causales physiques - cet adjectif étant plus générique que mécaniste - n'ont de valeur explicative qu'au niveau d'intégration déterminé par les caractères intentionnels du système individuel concerné et qu'en relation de subordination à ces intentionnalirés). Si les artefacts techniques sont d'abord dans 
l'environnement de celui qui s'apprête à les utiliser, comme on en a convenu ci-dessus, le fait de les placer sur la trajectoire des chaînes causales mentionnées comporte, dans sa complexité, au moins la relation d'action par laquelle l'artefact technique exerce obligatoirement l'influence (perturbation, contrainte, possibilité) spécifiée par ses propriétés inhérentes. Cette relation d'influence, je la représente par une relation causale (pas nécessairement simple, pas nécessairement linéaire) qui va de l'artefact au système individuel d'action et qui génère ainsi une nouvelle classe d'effets à inclure dans le cycle sans fin des impacts de la science et de la technologie.

On pourrait développer ici une typologie des effets dont on souhaite que le modèle formule le concept. Je ne suis pas prêt à proposer une telle typologie mais je suppose qu'il serait commode de distinguer, par exemple, entre les effets qui modifient le système utilisateur lui-même, soit au niveau de son activité d'idéation (notre point de départ), soit au niveau de la forme de son comportement, d'une part, et les effets observables sur les produits de l'utilisateur, d'autre part.

La nécessité de réidentifier chaque fois, c'est-à-dire à chaque stade du modèle où c'est pertinent, le système individuel impliqué vient naturellement du fait que ceux qui produisent des artefacts peuvent être distincts de ceux qui les utilisent ou consomment. Les fonctions et rôles sociaux des agents respectifs sont généralement différents.

Il peut sembler ici que c'est une tendance excessive à l'analyse qui me pousse à distinguer ici pour eux-mêmes, parmi les processus par lesquels un artefact de l'environnement technique engendre des effets propres à l'occasion de son utilisation, ceux qui affectent l'agent individuel ; car les objets techniques dont la présence est la plus massive et sollicite de ce fait plus fréquemment l'attention des chercheurs sont impliqués le plus souvent dans des processus de production définis à l'échelle des groupes (les organisations formelles par exemple, ou les professions, ou les travaux publics) et non dans des processus dont les paramètres sont définis au niveau de l'interaction avec un système individuel particulier ou avec un système individuel type (censé représenter plusieurs systèmes particuliers). 
Je reconnais aisément que le degré de pertinence des processus que je veux considérer sous la présente étape cinq du modèle peut varier considérablement d'un contexte de recherche à un autre; c'est même un fait, je crois, que la plupart des études d'impact, à l'heure actuelle, s'intéressent aux phénomènes qui ne se laissent percevoir que comme effet d'ensemble (productivité, taux de chômage, rapports capital-travail, qualité de vie, longévité, erc.), du genre de ceux que je considérerai à l'étape six du présent modèle. Néanmoins, je plaide pour que les relations d'impact susceptibles d'être définies au niveau de l'interaction entre le système individuel et l'artefact qu'il utilise soient identifiées le plus systématiquement possible. Non seulement ce qui se passe là a valeur de médiation pour bien des phénomènes d'ensemble établis sur un dénombrement des cas individuels mais il y a là un passage obligé pour l'observation de tous les processus qui concernent la production de représentations: car seuls des systèmes individuels produisent des représentations, et ils les produisent pour eux-mêmes avant (l'antériorité, ici, étant aussi bien chronologique que logique) qu'une quelconque généralisation permette de mettre en scène les représentations dites collectives.

Une problématique qui relève directement du stade que je suis en train de décrire est celle qui cherche à identifier si et dans quel sens l'utilisation d'artefacts techniques donnés affecte la manière de penser des utilisateurs. Une fois que l'on a bien précisé qu'il ne s'agit pas ici des transformations individuelles dues à l'information que l'utilisateur pourrait « lire » sur l'artefact, il est extrêmement délicat de préciser en quel sens l'interaction concernée affecte la manière de penser et de préciser la méthode permettant d'observer ces phénomènes. 1 y a d'abord à reconnaître la différence, masquée par l'appellation manière de penser, entre des formes de pensée et des contenus de pensée. Des hypothèses comme les suivantes concernent des formes de pensée: l'utilisation des ordinateurs entraîne chez l'utilisateur un développement préférentiel de la pensée analytique (ou analysante) par rapport à la pensée intuitive ou synthétisante; l'utilisation de tel artefact entraîne un développement préférentiel de la pensée par image relativement à la pensée par concepts, celle-ci étant considérée plus abstraite et plus articulée. Par contre, des hypothèses comme les suivantes concernent des contenus de pensée: l'utilisation régulière et 
exclusive d'une calculatrice provoque l'oubli progressif des tables de multiplication chez le sujet qui les connaissait d'abord; les possibilités offertes par les calculatrices (lorsque celles-ci sont considérées, par l'utilisateur, comme étant en principe accessibles) semblent être un facteur déterminant du fait que les utilisateurs, placés devant la possibilité de choisir, optent pour la stratégie recours à la calculatrice plutôt que pour la stratégie apprendre par coeur les tables de la multiplication. Je mentionne ces hypothèses pour illustrer la distinction conceptuelle entre forme de pensée et contenu de pensée, non pour suggérer qu'elles sont plausibles ou intéressantes; je crains au contraire qu'elles proviennent d'un regard spontané et naîf, au sens où les épistémologues utilisent ces termes pour désigner la pensée pré-scientifique. Mais déjà, à prendre au sérieux les problèmes que susciteraient la définition cohérente de leurs termes, l'explication poussée de leurs postulats, leur réfutation et leur corroboration éventuelles, on progresserait sans doute dans l'élaboration du cadre théorique qui fait présentement défaut.

Il y aurait également à définir des nuances appropriées entre des manières de penser et des attitudes ou dispositions; les manières de penser semblent être des caractéristiques de processus cognitifs et d'actes intellectuels tandis que les atritudes et dispositions sont des routines plus ou moins permanentes de comportements et relèvent en partie de l'émotivité de l'agent, en partie de ses contenus de pensée relativement sédimentés et en partie des seules habitudes (dont les plus immotivées sont peut-être les plus «pures »; «pures » au sens de: ne résultant de rien d'autre que de la répétition du comportement concerné).

Et la liste que j'improvise présentement pourrait se continuer avec ce troisième sous-problème : arrive-t-il, en général, et si oui comment, que le fait, pour un individu, de se comporter d'une certaine façon nouvelle pour lui induise chez lui une manière de penser nouvelle aussi? L'hypothèse selon laquelle des comportements induisent des représentations a désormais une sorte d'évidence à laquelle nous ont habitués des théories psychologiques comme celle de Piaget et des théories thérapeutiques pour lesquelles l'adoption de comportements nouveaux, dont on dit parfois qu'il s'agit de les expérimenter, permet au patient de sortir d'une névrose, d'un «double-bind» schizophrénique ou de je ne sais quoi 
d'autre. Or, il n'est justement pas certain qu'on puisse importer, en provenance de ces théories, quoi que ce soit d'utile pour le traitement de notre problème, ne fût-ce que pour les raisons suivantes: $1^{\circ}$ les expériences de Piaget n'ont jamais concerné la pensée adulte et, dans la mesure où elles ont mis en évidence des contenus de pensée, ce ne furent jamais que les contenus que j'appellerais primitifs, tels que les notions d'espace, de nombre, d'objet perceptible, etc, ; on n'a jamais su de quelles actionsmanipulations les croyances (celles des enfants aussi bien que des adultes) sont les schèmes, etc.; $2^{\circ}$ les psychothérapeutes, généralement, ne décrivent pas les guérisons de leurs patients en termes des manières de penser qui se succèdent en ces patients mais en termes de comportements (plus ou moins jugés selon leur valeur en regard de l'adaptation de l'individu à son milieu et selon leur capacité de générer du bien-être plutôt que de la souffrance); et quand ils s'intéressent aux représentations que se fait le patient, ce n'est jamais que sous l'angle de leur valeur symptomale et donc pratiquement jamais sous l'angle qu'on vise en parlant des manières de penser dans notre question initiale.

On peut partir, je pense, du fait qu'un artefact technique impose un certain nombre de comportements individuels, au titre de contraintes d'utilisation déterminées par lui-même, et en rend possibles un certain nombre (indéfini) d'autres, au titre de moyen pour réaliser des stratégies d'action déterminées complètement en dehors de lui-même (chaque utilisateur décide en fonction de ses objectifs professionnels par exemple, les tâches qu'il réalisera au moyen de l'ordinateur, du téléphone, des machines-outils, des produits chimiques disponibles, des matériaux artificiels, etc.). Or, à ce niveau, il y a, à première vue, peu de gestes, ou de suites de comportements qui exigent ou provoquent des formes de pensée ou des contenus de pensée déterminés, des croyances, des attitudes ou des dispositions déterminées; de sorte que le premier problème est de montrer qu'il y en a. J'ai cru, un jour, en écoutant un médecin vétérinaire formé en Allemagne fédérale raconter des événements de sa pratique au Pakistan, apercevoir intuitivement que certaines attitudes intellectuelles, certaines représentations de la mesurabilité et de la mesure du temps étaient effectivement des conditions préalables à un usage réussi des médicaments (qui sont bel et bien des artefacts) et des techniques d'insémination artificielle; et ces 
attitudes et représentations apparaissaient vaguement comme impliquées par, ou du moins liées à, une pratique (ou, si l'on veut, des structures de comportements) occidentale de la technique médicale parce qu'elles semblaient faire défaut chez les paysans auprès desquels les professionnels occidentaux étaient censés justement remplir des rôles d'assistance « technique ». Je ne sais si, en évoquant un tel exemple, je fais remonter trop loin le problème de l'identification de rapports entre utilisation d'artefacts techniques et manière de penser, ni si je fais dévier indûment le problème initial vers le sous-problème - qui n'est pas vraiment le nôtre des rapports entre utilisation d'un artefact technique et connaissances préalables (pensons, par exemple, aux connaissances des commandes que doit posséder un usager de l'ordinateur pour faire du traitement de texte). En tout cas, l'exemple suggère que certaines manières de penser n'apparaîtront comme spécifiquement liées à l'utilisation d'artefacts techniques qu'à la faveur d'un contraste avec des situations desquelles elles seront absentes, ces situations devant être définies elles aussi par des relations entre comportements et manières de penser.

Il me semble présentement très difficile d'établir sur une base empirique relativement intersubjective l'une ou l'autre hypothèse concernant la manière dont l'utilisation d'artefacts techniques affecte la pensée des individus et même leurs attitudes. Mais ce sont bel et bien de telles hypothèses qu'il faut néanmoins établir, à mon avis, car sans elles les hypothèses concernant les groupes, y compris la "société post-moderne" (cette entité parfois tant aimée) n'auront aucune valeur scientifique: l'un parlera de la technologie comme causant une recrudescence du scientisme, l'autre comme causant une dévalorisation du politique, l'autre encore comme gauchissant la représentation que l'homme se fait de son rapport à la nature, ou même (pourquoi pas?) de son rapport avec Dieu. Pour moi, ces thèses sont l'expression presque directe de certaines visions du monde, ou du moins de présupposés philosophiques. Et il sera plus difficile de montrer comment une vision du monde s'installe chez un individu à l'occasion et à cause de l'utilisation d'artefacts techniques que de montrer que la vision du monde adoptée par certains auteurs se loge dans les thèses qu'ils avancent au sujet de l'impact social de la science et de la technologie. 


\subsection{L'environnement externe $\mathrm{E}_{\mathrm{x}}$ comme terme-source de relations causales «naturelles »}

Si une bombe atomique explose en un certain endroit et tue tous les humains qui se trouvaient à au moins $15 \mathrm{~km}$ de cet endroit, on peut sans doute considérer qu'il s'agit d'un cas où un artefact technique, de même que la science qui en a rendu possible la réalisation, a eu un impact social. De fait, du point de vue du modèle que je construis, cet exemple appartient à la phase six dont je vais traiter ci-après, mais il illustre d'emblée ce que je veux entendre par des relations causales « naturelles ». Il s'agit maintenant de ramener la notion de causalité naturelle aux cas où elle joue dans l'interaction entre une système individuel et la nature.

(Celui qui a lancé la bombe, sans en subir les effets destructeurs naturels, a modifié l'environnement d'un grand nombre de systèmes individuels, et si l'on pense que son activité est à quelque titre une activité techno-scientifique, de sorte qu'elle tombe sous la catégorie des objets que le présent modèle entend décrire, alors la relation d'impact dont son action est le terme-source est à décrire au moyen des catégories de la phase deux du modèle, à savoir : La production émanant de $S I_{n}$ ).

Comme je l'ai annoncé dans l'introduction à la section cinq, juste avant de commencer la section 5.1, le type d'impact que je vais maintenant décrire en 5.3 concerne des « actions » dont l'initiative appartient à la nature plutôt qu'au système d'action individuel. Le concept non technique d'initiative requiert ici quelque précision.

Les systèmes individuels sont conçus ici, par définition, comme des systèmes actifs, c'est-à-dire comme possédant en euxmêmes le principe de leur action; une spécification de ce concept de système actif est donnée par le concept d'autonomie, au sens de Varela et Maturana; pour ces auteurs, les systèmes inanimés ne sont pas autonomes, les systèmes vivants le sont sûrement et c'est ce qui les caractérise comme vivants; les systèmes sociaux le sont peut-être mais il faudra le montrer (Varela espère pouvoir le montrer). Pour moi, certains systèmes inanimés sont actifs (par exemple, le système planétaire, eu égard à ses mouvements, donc du point de vue de la mécanique céleste ; autre exemple : le système formé par un atome radioactif) ; les systèmes vivants sont sûrement actifs; les systèmes sociaux ne le sont pas (mais comme je l'ai dit 
plus haut, dans la section 3, je n'en fais pas une thèse). Quand je dis qu'un système individuel a l'initiative d'une action, j'entends qu'il agit en tant que système intentionnel (la plupart des animaux sont incapables d'intentionnalité, mais comme je ne suis pas en mesure de préciser toutes les conditions d'application de ce concept, je laisse ouverte la possibilité que certains animaux supérieurs soient capables d'intentionnalité ; les systèmes qui ne sont pas actifs sont incapables d'intentionnalité, donc les groupes sociaux, y compris les sociétés sont incapables d'intentionnalité (exemple : les régularités découvertes par Durkheim concernant les taux de suicide sont des faits sociaux, mais ne résultent pas d'un comportement intentionnel, comme le serait un comportement décisionnel, de la société). Quand je dis que la nature a l'initiative d'une action, je veux dire que le changement qui survient dans le système individuel (actif, autonome, toujours) n'est pas déterminé par l'intentionnalité du système individuel ; un système individuel n'agit pas toujours en tant que système intentionnel, il agit parfois seulement comme système naturel ; il n'agit jamais seulement comme système intentionnel; quand il agit en tant que système intentionnel, il ne peut se soustraire à sa condition de système naturel.

À la présente étape du modèle, il s'agit de décrire des processus par lesquels des objets ou états de choses en position d'environnement affectent des systèmes d'action individuels; plus explicitement, les situations à décrire satisferont les trois conditions suivantes:

- que la cause concernée soit due à l'activité technoscientifique;

- qu'il y ait des effets sur des systèmes d'action individuels ;

- que la relation causale soit naturelle.

La satisfaction de chacune de ces conditions pose divers problèmes, qui gagnent en complexité dès que l'on quitte les cas relativement clairs sur lesquels la pensée journalistique, portant des jugements simples, semble avoir concentré l'attention et l'émotion (par exemple, cas de pollution de l'environnement par des produits toxiques ou radioactifs; cas des merveilles de la technologie médicale à fonction thérapeutique). 


\subsubsection{La distinction entre activité techno-scientifique et activités d'une autre sorte}

Dans les disciplines anthropologiques, où il est pertinent d'opposer nature et culture, la notion d'activité technique en vient souvent à être pratiquement coextensive à la notion d'activité humaine ; et l'on utilise souvent, pour faire comprendre le propre de la culture, l'idée que l'être humain impose une forme (de son choix, pour ainsi dire) aux objets ou états de la nature, y compris aux éléments de sa propre nature comme individu vivant (par exemple, une forme régissant des comportements ordonnés à satisfaire des besoins). Cette apparition des formes est associée de façon plus ou moins complexe (dirai-je aussi : confuse?) à l'apparition du sens, pour la distinguer plus ou moins nettement de l'apparition des formes imposées à la nature par certains animaux. Les artefacts sont les objets matériels ayant reçu une forme par le fait de l'intervention humaine; l'anthropologie sait toujours les distinguer, paraît-il, des produits «formés» par les animaux; et pas toujours, disent certains, des produits «formés» par les visiteurs extra-terrestres. L'apparition de l'artefact marque l'apparition de la culture, celle-ci devenant l'objet de la culturologie (terme que préférait Lewis White) ou de l'anthropologie culturelle (comme disent la plupart des Américains) ou de l'ethnologie.

Nous convevons tous qu'en parlant aujourd'hui d'activité techno-scientifique, puis de science et technologie, nous désignons autre chose que la production d'artefacts au sens anthropologique. Cependant la conceptualité, ici, n'est pas claire. Considérons, par exemple, les cas d'activités très anciennes ayant toujours transformé l'environnement et dont la manière de transformer l'environnement a été affectée sensiblement par des innovations techniques, à certains moments de l'histoire : l'agriculture, la construction d'édifices, les soins de santé. Les problèmes que j'entrevois rapidement sont comme les suivants. (P1 :) À partir de quel seuil d'activité un état donné de l'environnement (état que nous nous apprêtons à mettre en position de cause) est-il imputable à la science et à la technologie au sens «moderne " ? La définition du concept de seuil exigera de faire intervenir plusieurs variables dont certaines seront sans doute étonnantes. (P2:) Pour un état donné de l'environnement, quels effets (sur les systèmes d'action) seront imputables aux résultats cumulés des activités pré-technologiques et lesquels 
seront imputables spécifiquement aux activités technologiques? L'activité et la prodigalité des sols ont été modifiées par des activités de niveau pré-technologique et, pour des segments donnés d'espace-temps, ces modifications ont des effets cumulatifs. (On dit que l'île de Pâques a finalement succombé à l'érosion suite à un élevage trop intensif de moutons). La croissance des villes est un processus éminemment cumulatif : sur les individus habitant une ville s'exercent vraisemblablement des influences composites, à proportion que se trouve composite l'état lui-même de cet environnement; quelles influences seront imputables aux aspects de l'état (représentés dans la théorie par des grandeurs d'états, ou variables d'état) produits par l'activité techno-scientifique et, par ce biais, à l'activité techno-scientifique elle-même?

Peut-être y a-t-il lieu de formuler le problème à un niveau de généralité plus grand. L'environnement des systèmes d'action, à quelqu'univers de pertinence qu'on veuille bien le limiter pour les fins d'un contexte de recherche, a une histoire; cette hypothèse simple (que je crois vraie) a pour conséquence que l'état de cet environnement est composite, on dira parfois sédimenté, et que les composantes techno-scientifiques ne sont jamais que parmi les plus récentes, si tant est qu'on donne un sens contemporain à technologie et à science; selon la métaphore de la sédimentation, la composante techno-scientifique sera la plus superficielle. Dans ces termes, le problème est donc d'identifier cette composante dans le temps, en justifiant l'identification par un réseau conceptuel plus ou moins fin, mais explicite, comportant, d'une part, une périodisation et, d'autre part, une hiérarchie de niveaux d'invariance temporelle.

En deuxième lieu, l'environnement des systèmes d'action résulte de toutes les activités de ceux-ci, du moins de toutes celles dont les produits parviennent à exister dans l'environnement, c'est-à-dire à marquer d'une forme, la leur, un fragment de l'univers physique, cette notion de forme étant celle que j'imputais tout à l'heure aux anthropologues théoriciens de la culture. Or, les systèmes d'action ont d'autres activités que l'activité technoscientifique; celui qui étudie l'impact (social) de la science et de la technologie est obligé, il me semble, de faire cette hypothèse. Dans ces termes, le problème est donc d'identifier la composante techno-scientifique de l'environnement, non plus dans le temps, 
mais dans l'espace conceptuel (structural) où le chercheur étale sa typologie des activités humaines qu'il croit devoir distinguer entre elles pour circonscrire son propre objet.

\subsubsection{Le problème de l'existence même d'effets repérables sur des systèmes individuels}

En conséquence des postulats que jaai admis jusqu' ici concernant le caractère actif des systèmes d'action individuels et le caractère non-actif des systèmes d'action sociaux, je pose ce que je vais appeler le postulat de la priorité ontologique des réactions individuelles par rapport aux réactions collectives, à savoir : pour qu'il y ait des effets d'ensemble, il faut qu'il y ait au préalable des effets sur des systèmes individuels.

Ce postulat concerne le sens de la relation causale dans le processus objectif; de ce fait, c'est un postulat ontologique. Il n'exclut pas cependant que, du point de vue (épistémologique) de la progression de la connaissance de la relation causale, la découverte, la perception ou l'observation d'une relation causale n'aient lieu, de fait et le plus souvent, que lorsque les effets sur les systèmes individuels ont déjà pris des proportions d'ensemble qui en augmentent à la fois la visibilité et la plausibilité. Le postulat de la priorité ontologique des réactions individuelles n'exclut pas non plus, à mon avis, les thèses qui affirment la priorité ontologique de la société et de la culture (par exemple) sur l'individu et qui veulent exprimer par là le fait que, dans tous les cas qu'il est possible à un chercheur d'observer, en matière de comportements individuels, ceux-ci sont toujours redevables d'une ontogenèse qui s'est déroulée dans un environnement comportant déjà une société et une culture constituées, notamment un langage. Bien sûr, l'individu arrive dans un monde déjà là, mais il est constitutionnellement autonome. Ce sont les autonomies qui constituent les faits d'ensemble. Cela vaut pour tous les être vivants ; cela vaut pour les faits d'ensemble collectivement dénommés la convention sociale.

Les cas-types et faciles dans lesquels on parle à juste titre d'impacts naturels exercés par la science et la technologie sur les individus sont ceux qui concernent l'apparition, dans l'environnement biologique, de produits auxquels réagissent les organismes des 
individus; ces produits sont considérés tantôt comme nocifs (par exemple, l'anhydride sulfureux), tantôt comme bénéfiques (par exemple, la chaleur conférée aux espaces habités par des moyens technologiques ; cependant, pour que cet exemple n'entre pas dans la catégorie des actes intentionnels d'utilisation d'un item de l'environnement, je le restreins aux cas où l'individu qui bénéficie de la chaleur n'est pas celui qui en a contrôlé la production. J'aurais aimé trouver un exemple dans lequel le produit soit une substance, au sens chimique du terme, et soit considéré comme bénéfique sans être de ceux qu'on s'approprie dans un comportement intentionnel d'utilisation; je n'y parviens pas. Peut-être le Hollandais habitant un «polder » bénéficie-t-il d'un item de l'environnement, créé technologiquement par d'autres, sans se l'approprier davantage qu'un Montréalais ne s'approprie l'anhydrire sulfureux de l'air qu'il respire...).

Il y a cependant une exploration à faire de cas moins clairs ; je pense à deux sortes de difficultés :

a) on est en présence de composantes techno-scientifiques flagrantes de l'environnement et on ne leur trouve pas d'effets spécifiques et naturels sur les systèmes individuels ;

b) on résiste à l'idée qu'un trait techno-scientifiquement généré de l'environnement physico-biologique n'ait pas d'effet naturel sur les systèmes individuels et on se demande si cela est possible, théoriquement acceptable, provisoirement avouable.

Le chercheur intéressé à découvrir les impacts de la science et de la technologie sur les humains souhaite tout naturellement découvrir des impacts et les dévoiler. En dehors des cas faciles, il est aux prises avec les deux difficultés mentionnées. Affrontant la première, il doit découvrir une chaîne de médiations depuis l'item environnemental jusqu'à l'état du système individuel. La chaîne des effets naturels impliquant des lois de la nature peut passer par la couche d'ozone atmosphérique avant d'atteindre un organisme vivant. Les chaînes causales du genre «Avec cet instrument, il sera possible de déplacer tant de tonnes de roc en tant de minutes - ce qui permettra de construire tel barrage - ce qui permettra de produire tant d'électricité - ce qui permettra de... » sont de longueur variable, et parfois très longues, et peuvent ne concerner 
que des transformations de systèmes naturels, selon les régularités énoncées dans les lois des sciences de la nature. Le modèle que je suis en train d'esquisser suggère de penser à identifier à quelle occasion (à l'occasion de quelle relation causale), et à quel moment de la chaîne, un certain trait de l'environnement entraîne une modification du système individuel, c'est-à-dire constitue l'élément, ou un élément, qui déclenche la réaction du système. C'est à ce moment seulement que la relation d'impact entre à nouveau dans la zone des systèmes d'action.

Affrontant la deuxième difficulté, à savoir la résistance à l'hypothèse d'un non-effet, le chercheur aura l'occasion de poser quelques questions fondamentales concernant la relation de causalité, laquelle reste l'une des plus fécondes sources de «puzzles» philosophiques, en théorie de la science et en théorie de la connaissance. L'hypothèse selon laquelle un trait de l'environnement est sans effet naturel sur un système individuel, et même sur la plupart des systèmes individuels en présence, est-elle plus étonnante que l'hypothèse contraire affirmant que le trait en question a un effer, un impact comme on dit? Dans la profusion des discours qui ont plutôt tendance à renchérir à propos des impacts, il me plaît assez d'alléguer que la classe des composantes techno-scientifiques de l'environnement qui n'ont pas d'impact naturel sur les individus est plus étendue que ne le donnent à penser les études d'impacts. Que l'environnement urbain, ou celui, plus restreint, de l'habitation, soit de pierre, de terre cuite, de crépi, de ciment, de brique ou de verre, aucune de ces variations n'introduit en tant que telle de modification correspondante dans le système individuel considéré comme système naturel, lorsque les effets naturels de l'une sont technologiquement compensés pour équivaloir, justement, aux effets naturels de l'autre (et les techniques de contrôle de l'environnement habité réalisent presque cette «équivalence » pour des variables telles que la quantité de lumière, la température, le degré d'humidité relative, etc.). Le fait que le plastique remplace l'acier ou le bois dans la fabrication d'un grand nombre d'objets d'usage courant a nécessairement pour effet qu'il y a plus de plastique dans l'environnement en 1987 qu'en 1950 ; et cela est d'autant plus vrai que le plastique n'est pas biodégradable. Cette modification de l'environnement modifie-t-elle à son tour les systèmes individuels ? Je ne connais pas d'impact, dans ce cas, qui soit à la fois direct et 
naturel. Je crois qu'il en est ainsi pour la plus grande part des matières qui apparaissent dans l'environnement bio-physique comme produits de l'activité techno-scientifique.

La formulation de la dernière phrase ci-dessus me suggère un niveau de généralité assez élevé qu'on peut adopter pour formuler les hypothèses sur l'existence d'effets naturels. L'acrivité technoscientifique introduit trois types de modifications de l'environnement naturel :

$\mathbf{M}_{1}$ : elle change la répartition naturelle de l'énergie, surtout en créant des sources (actuelles ou potentielles) d'énergie et des moyens de contrôler la répartition d'énergie ;

$\mathrm{M}_{2}$ : elle change la composition naturelle et la répartition naturelle de la matière, surtout en créant des matériaux (dont certains existent déjà naturellement, d'autres non) et en transportant de la matière d'un lieu à un autre ;

$\mathbf{M}_{3}$ : elle change les quantités et les contenus d'information inscrits dans l'environnement, surtout du fait qu'elle en produit, emmagasine et véhicule.

Or, les modifications $M_{3}$ ne sont jamais causales au sens naturel. Les modifications $\mathrm{M}_{1}$ et $\mathrm{M}_{2}$ sont, pour la plupart, de celles qui entrent en relation causale avec le système individuel à l'occasion d'une urilisation-appropriation de la part de l'individu, ce qui a pour effet d'introduire dans le processus causal des éléments intentionnels. Il reste dans $M_{1}$ une sous-classe de modifications dont le système individuel subit des effets sans s'engager dans un processus d'utilisation-appropriation (on pourrait sans doute également faire jouer ici le concept de consommation); je conjecture que ces modifications sont toujours à effet négatif (la chaleur dont bénéficie le client des édifices publics, comme dans mon exemple ci-dessus, n'est pas vraiment une énergie seulement subie et non-appropriée car elle est quelque part, au bout du compte, payée par le bénéficiaire; il y a, dans la chaîne des processus, un élément intentionnel même s'il est différé). De même une sous-classe de $\mathrm{M}_{2}$, complémentaire de la classe principale, comprend des modifications non susceptibles d'être recherchées par un système individuel. La différenciation et la définition des 
environnements dans lesquels ces modifications sont susceptibles d'exercer des effets sont compliquées et me semblent de fait peu développées, dans l'état actuel des études d'impact. L'environnement dont on entend le plus souvent parler est l'environnement biologique, dans le contexte d'une préoccupation pour la santé du système individuel. En regard de cet environnement, le fait qu'un matériau soit bénéfique le place presqu'automatiquement dans la catégorie des objets recherchés, donc dans la classe principale; le fait qu'il soit nocif ou indifférent le place dans la sous-classe complémentaire des modifications seulement subies. Je conjecture, dans ce contexte, que dire d'une modification qu'elle est indifférente, eu égard à la santé du système individuel, c'est dire simplement qu'elle n'a pas d'effet repérable. Quant aux modifications nocives, elles ont évidemment des effets qui peuvent être décrits dans le langage des processus régis par des lois de la nature. Cette sousclasse est finalement peu étendue, il me semble, et je conjecture qu'on peut lui appliquer une dichotomie très simple qui la divise exhaustivement en deux : $1^{\circ}$ les modifications voulues par l'activité techno-scientifique, et alors celle-ci est belligérante $; 2^{\circ}$ les modifications non voulues, et alors ces modifications ont un caractère résiduel.

\subsubsection{Les lois de la nature et l'autonomie des systèmes individuels}

Il s'agit, dans la section 5.3, des impacts exercés par l'environnement sur des systèmes individuels et en vertu d'un relation causale naturelle. Un problème qui pourra se poser est celui de concilier le déterminisme nomique avec le caractère autonome des systèmes individuels. Devant une donnée de l'environnement naturel, les réactions des systèmes individuels manifesteront quelques différences, dont la distribution constitue la variabilité associée à cette relation causale. Du point de vue de la clarification des concepts, il est important de noter que la variabilité des réactions, telle qu'elle est entendue ici, n'est pas celle introduite par les états intentionnels des systèmes individuels. L'arrière-fond théorique évoqué ici est la théorie de l'autonomie de l'être vivant, telle que développée par Humberto Maturana et Francisco Varela.

Il est possible, donc, que la relation causale, même si elle est naturelle, soit indéterministe en un sens légèrement différent de 
l'indéterminisme purement statistique. Des études d'impact soucieuses de rigueur théorique expliciteront le type de variabilité qui relativise la relation causale et, si possible, les facteurs présumés qui font varier les réactions individuelles. Si le chercheur joint à cette donnée l'explicitation habituelle des limites d'applicabilité de la relation causale exhibée, il aura établi pour elle ce que l'on pourrait appeler son profil de variabilité-généralité.

Naturellement, l'un des facteurs de variabilité qui va constamment mettre en cause le caractère naturel de la relation causale est la culture. J'en traiterai dans la section qui suit.

\section{L'amplification socioficatrice des réactions individuelles à l'environnement techno-modifié}

Les relations d'action décrites à la section 5, relations par lesquelles l'impact provient de l'environnement et affecte les systèmes d'action individuels, se multiplient à proportion que les « espaces» techno-modifiés sont vastes, à proportion du nombre d'individus réagissants, à proportion, en fin de compte, du nombre des activités individuelles affectées par des contraintes, possibilités et perturbations de l'environnement.

Le principe quantitatif de l'amplification socioficatrice joue, dans cette portion du cycle des impacts, de la même manière que dans la portion qui traitait ci-dessus de l'amplification des impacts individuels sur l'environnement. Il n'est pas nécessaire que j'y revienne.

Pour établir une typologie sommaire des processus d'amplification, on peut reprendre chacun des trois types d'impact de l'environnement distingués dans la section 5 ci-dessus et leur faire correspondre un type de généralisation:

- généralisation des processus cognitifs par lesquels un système individuel retire de l'information de ses divers environnements ;

- généralisation de la réponse individuelle à l'artefact technique, principalement sous la forme de l'utilisation de ces artefacts par des décideurs ; ce qui implique que les artefacts 
sont intégrés à des chaînes intentionnelles qui les asservissent à des fins, motifs ou intérêts dont le décideur est à la fois le siège et l'origine ;

- généralisation des réactions naturelles des systèmes individuels aux données de l'environnement techno-modifié, dans les cas où les réactions procèdent selon les régularités assimilables à des lois de la nature.

La classification conceptuelle des relations d'impact impliquées dans ces processus de généralisation rencontre ici, à un degré plus élevé peut-être qu'en aucun autre segment du modèle, le problème de la différenciation entre les conditions socio-culturelles (qu'on peut considérer comme pré-technoscientifiques ou para-technoscientifiques) de la réaction imputable aux systèmes d'action, d'une part, et, d'autre part, les conditions plus spécifiquement technoscientifiques. Car, comme il a été mentionné précédemment, les systèmes individuels concrets sont toujours à quelque degré les dépositaires des influences socio-culturelles qui les ont formés et réagissent habituellement en tant que membres d'un système d'action déjà collectif, même si la réaction des individus, et elle seule, contribue elle-même en retour, une fois "amplifiée », à infléchir l'état même des croyances, dispositions, savoir-faire et attitudes qui constituent conjointement les composantes proprement culturelles (au sens de Parsons, notamment) des environnements collectifs (partagés) des systèmes individuels.

Un des points importants de la théorisation des relations d'impact est l'analyse conceptuelle de la notion cybernétique de contrôle; car la fonction contrôle impliquée dans la détermination des actitivés de systèmes individuels autonomes est à « répartir ", pour ainsi dire, entre des éléments de l'environnement externe des systèmes d'action et des éléments de l'environnement interne (au sens d'intériorisé) des mêmes systèmes. Les approches herméneutiques et dialectiques, celles notamment de la sociologie "compréhensive », privilégient les éléments de l'environnement interne (le privilège pouvant aller jusqu’à être exclusif), tandis que les approches objectivistes ou "positivistes" de la sociologie préconisant les méthodes quantitatives et les modèles prédictifs semblent privilégier les éléments de l'environnement externe. Dans un cas comme dans l'autre, je pense, la théorie ne réussit pas 
à intégrer de façon rigoureuse, dans la définition de la norion de contrôle, un concept opérationnel d'information, capable de servir aussi bien à la description de l'activité de systèmes sociaux qu'à celle de systèmes individuels.

\section{III}

\section{QUALITÉS, LIMITES ET LACUNES DU PRÉSENT MODÈLE \\ (Conclusion)}

Sans prétendre résumer systématiquement le présent modèle, je le livre à l'examen et à la pratique des chercheurs en soulignant quelques-unes des idées de base qui ont inspiré sa conception.

Premièrement, le présent modèle invite à concevoir les relations d'impact comme susceptibles de décompositions nombreuses et comme toujours impliquées dans des cycles. Les plus "grands" de ces cycles sont ceux par lesquels on représente les impacts (sur des environnements, au pluriel) des activités des systèmes d'action ainsi que les impacts en retour des environnements sur les activités des systèmes d'action, les mêmes ou d'autres. En conséquence, c'est toujours en vertu d'une simplification, même si celle-ci est habituellement nécessaire, que l'on représentera les relations d'impact par des flèches unissant des termes-sources à des termes-cibles; non seulement les causalités s'exerçant dans un sens sont pratiquement toujours doublées par des causalités en retour (chaque action est susceptible d'une rétroaction) mais encore chaque relation d'impact est "composable " avec d'autres (en d'autres mots, la causalité n'est généralement pas linéaire) et décomposable en plusieurs autres (en d'autres mots la causalité est généralement complexe, tant par ses termes que par les relations entre eux).

Deuxièmement, le présent modèle propose une façon d'articuler conceptuellement les notions de système d'action individuel et système d'action collectif (l'adjectif social convenant parfois, et parfois non). L'articulation repose sur certains postulats que $j$ 'ai explicités (par exemple, à l'occasion des définitions des termes actifs, autonome, intentionnel) et sur certaines intuitions que j'ai évoquées, sans parvenir à les expliciter, dans des postulats spécifiques (par exemple, l'intuition que le système d'action collectif dont un 
individu est membre n'est pas dans une relation "d'extériorité ", par rapport à ses membres composants, du moins pas dans la relation d'extériorité qui lie un membre composant à un autre du même collectif, ni dans la relation d'extériorité qui relie un système individuel à l'environnement dans lesquels s'inscrivent certains de ses produits, lorsque ceux-ci acquièrent l'existence autonome, ou du moins inter-subjective, de causes possibles pour l'activité d'autrui).

Ceci dit, il reste que ce modèle est purement conceptuel en ce sens qu'il s'adonne seulement à la clarification de concepts; il n'organise pour l'instant aucun ensemble de données empiriques. Cette caractéristique n'est guère surprenante pour un modèle conçu par un professionnel de la philosophie; si donc un tel modèle est utile, je n'anticipe pas que ce soit à un autre niveau que celui de la clarification conceptuelle. Du point de vue du chercheur aux prises avec la cueillette et l'interprétation de données empiriques, la généralité du discours philosophique en constitue une limite importante, et je tiens à dire ici que je reconnais cette limite. Par ailleurs, comme le «social scientist» se préoccupe et s'occupe, bien souvent, de remettre en question ou simplement de clarifier les concepts au moyen desquels il organise et interprète ses données empiriques, le travail du philosophe, malgré ses évidentes limites, peut devenir pertinent ou stimulant.

Le modèle possède également une limite importante, plus notoire que la précédente, de l'ordre de la lacune : il ne présente pas de moyens conceptuels pour résoudre le problème, mentionné pourtant à quelques reprises, de la différenciation entre les chaînes causales déclenchées par l'activité techno-scientifique et ses produits, d'une part, et les chaînes causales déclenchées par d'autres types d'activités, celles, notamment, qu'on a coutume de qualifier d'économiques, politiques et culturelles. C'est un vieux cheval de bataille, dans les disciplines préoccupées par les impacts de l'activité techno-scientifique, que la question de savoir si les produits des sciences et des techniques sont d'avance et déjà marqués par des partis-pris économiques, politiques et culturels, ou s'ils sont ainsi marqués, après coup seulement, en vertu d'une récupération qui les intègre à des chaînes intentionnelles tout à fait étrangères aux activités dont ils sont les résultats. La thèse selon laquelle l'utilisation 
des artefacts techniques a pour effet d'amplifier (ou plus fortement : "a pour effet seulement d'amplifier») les traits qui caractérisent déjà les pratiques sociales, en particulier les pratiques de production et les rapports sociaux de production, est une dérivée intéressante de l'une de deux options évoquées plus haut à savoir celle-ci: l'activité techno-scientifique, loin d'être neutre à l'égard des activités économiques, politiques et culturelles qui pourraient en récupérer les produits a posteriori, est déterminée par les conditions économiques, politiques et culturelles de sa propre occurrence d'une manière telle que ses propres effets vont dans le même sens que ceux des activités non techno-scientifiques mentionnées (rétroaction positive, et non négative).

Ce passionnant problème me semble au cœur des recherches concernant l'impact social de la science et de la technologie. Le modèle que je viens de proposer ne le résoud certes pas, mais je crois qu'il contient quelques outils conceptuels capables de contribuer à le mieux poser. Si d'autres chercheurs lui consentent un peu ce mérite, il aura rempli son office.

Département de philosophie

Université du Québec à Montréal

\section{LECTURES COMPLÉMENTAIRES}

BENNETT, R.J. et R.J. CHORLEY, Environmental Systems : Philosophy, Analysis and Control, Princeton, Princeton University Press, 1978.

MATURANA, Humberto R. et Francisco J. VARELA, Autopoiesis and Cognition. The Realization of the Living with a preface by Sir Stafford Beer. Dordrecht et Boston, D. Reidel (coll. "Boston Studies in the Philosophy of Science », vol. 42), $1980 ; 141 \mathrm{p}$.

NORA, Simon et Alain MINC, The Computerization of Society, avec une introduction de Daniel Bell. Cambridge (Mass.), The M.I.T. Press, 1980.

ROPOHL, Günther, “A critique of technological determinism », pp. 83-96 dans: DURBIN, Paul T. et Friedrich RAPP, dir. publ., Philosophy and Technology, Dordrecht et Boston, D. Reidel (coll. «Boston Studies in the Philosophy of Science », vol. 80), 1983.

SOUZA, G.R. de, System Methods for Socioeconomic and Environmental Analysis, Lexington (Mass.), D.C. Heath \& Co., 1979. 
VARELA, Francisco J., Principles of Biological Autonomy. New-York et Oxford, Elsevier North-Holland (coll. "General Systems Research », vol. 2), 1979 ; $306 \mathrm{p}$.

VICKERS, Sir Geoffrey, "The ecology of ideas", in: Value Systems and Social Process, New-York, Basic Books, 1968.

WOJCIECHOWSKI, Jerzy A. "Man and knowledge: one or two systems?", PP. 427-38 dans : BANATHY, Bela, dir. publ., Systems, Science and Man, Louisville (Ky), 1980.

, "The impact of knowledge on man: the ecology of knowledge", pp. 161-75 dans: Hommage à François Meyer, Marseille, Publications de l'Université de Provence, 1983. 